\section{Continuing posterior tibial nerve stimulation after twelve weekly sessions: a randomized controlled trial}

\author{
Jessica S. Zigman, ${ }^{1}$ Stephanie J \\ Handler, ${ }^{2}$ Stephanie Amaya, ${ }^{3}$ Aldene $O$ \\ Zeno, ${ }^{4}$ Yoko Takashima, ${ }^{5}$ John J Navas, ${ }^{6}$ \\ Tajnoos Yazdany?
}

${ }^{1}$ Palo Alto Medical Foundation,

Mountain View, CA; ${ }^{2}$ Division of Female

Pelvic Medicine and Reconstructive

Surgery, Department of Obstetrics and

Gynecology, University of California,

Riverside School of Medicine, Riverside,

CA; ${ }^{3}$ Department of Psychology,

University of Wyoming, Laramie, WY;

${ }^{4}$ Division of Female Pelvic Medicine and

Reconstructive Surgery, Department of

Obstetrics and Gynecology, Keck School

of Medicine of USC, Los Angeles, CA;

${ }^{5}$ St. Jude Heritage Medical Group,

Fullerton, CA; ${ }^{6}$ Penn Medicine

Lancaster General Health, Lancaster,

PA, USA; ${ }^{7}$ Division of Female Pelvic

Medicine and Reconstructive Surgery,

Department of Obstetrics and

Gynecology, Harbor-UCLA Medical

Center, Torrance, $\mathbf{C A}$

\begin{abstract}
Posterior Tibial Nerve Stimulation (PTNS) is a treatment option for refractory Overactive Bladder (OAB). There is currently no evidence to support which maintenance schedule is most effective. The objective of this study is to compare patientreported outcomes between monthly maintenance therapy and sessions scheduled Per patient request (PRN). We hypothesized that there is no difference in these measures between groups. After completing 12 sessions of PTNS, patients were randomized to monthly or PRN maintenance. Quality of life, patient satisfaction, and degree of symptom bother were assessed with validated questionnaires. Thirty six patients were randomized: 19 to monthly follow-up, and 17 to PRN follow-up. The PRN group demonstrated a significantly higher quality of life $(\mathrm{p}=0.04)$ with a large effect size (0.82). Over a three-month period, there was no significant difference in the average number of visits between the monthly and PRN groups, with an average of 1.79 $( \pm 1.18)$ and $1.24( \pm 1.35)$ visits, respectively $(p=0.20)$. After six months of maintenance therapy, $42 \%$ of patients in the monthly
\end{abstract}

group and $53 \%$ of patients in PRN group elected to continue therapy $(p=0.27)$. There was no significant difference between the groups with regard to treatment satisfaction $(p=0.62)$ or percent change in OAB symptoms $(p=0.13)$. There is no difference between monthly and PRN follow-up in terms of patient satisfaction and symptom bother. However, the PRN group scored higher on the quality of life measure. PRN maintenance may optimize care for patients treating Overactive Bladder Syndrome with posterior tibial nerve stimulation.

\section{Introduction}

Overactive Bladder Syndrome (OAB) affects millions of adults, with profound personal and economic costs. Several management options exist for $\mathrm{OAB}$, including conservative treatment, pharmacotherapy (mainly antimuscarinic drugs), intravesical injection of Onabotulinum toxin A, or procedural treatments aimed at modulating bladder reflex pathways, such as Posterior Tibial Nerve Stimulation (PTNS) or Sacral Nerve Stimulation (SNS). ${ }^{1}$

Although antimuscarinic drugs can cause a reduction in $\mathrm{OAB}$ symptoms, the effect is modest, and many patients are intolerant of the side effects. ${ }^{2}$ Along with intravesical injection of onabotulinumtoxinA, neuromodulation of bladder reflex pathways via either PTNS or implanted SNS has been acknowledged as the next step in the algorithm of care. ${ }^{3}$ PTNS provides an option for patients whose OAB symptoms are refractory to anticholinergic therapy, and is less invasive and less costly than SNS. ${ }^{4}$ PTNS has been wellstudied and is theorized to inhibit contractions of the detrusor muscle, reducing urinary frequency and urgency. ${ }^{5,6}$ Furthermore, a Cochrane review concluded that PTNS was more effective than both no treatment and drug treatment for OAB. ${ }^{1}$

Although PTNS is uniformly administered via 12 weekly treatment sessions, there are no studies comparing different PTNS maintenance treatment regimens, although the STEP study [new citation here, previously was ${ }^{19}$ followed a single tapering protocol. Findings from our study can optimize care for patients with $\mathrm{OAB}$, as PTNS maintenance can be costly and time-intensive. ${ }^{1}$ Additionally, the literature suggests that assessing patient-reported outcomes such as quality of life and patient satisfaction should constitute an integral part of treatment efficacy assessment..$^{7-9}$ However, there is a paucity of research investigating subjective outcomes of treating $\mathrm{OAB}$ with PTNS.

The objective of this study is to com-
Correspondence: Stephanie J. Handler, Division of Female Pelvic Medicine and Reconstructive Surgery, Department of Obstetrics and Gynecology, University of California, Riverside School of Medicine, 19330 Jesse Lane Suite 100, Riverside, CA 92508, USA.

E-mail: stephanie.handler@medsch.ucr.edu

Key words: Overactive bladder; posterior tibial nerve stimulation; percutaneous tibial nerve stimulation; maintenance treatment; urgency urinary incontinence.

Acknowledgements: This work was presented as a short oral presentation at the American Urogynecologic Society's Pelvic Floor Disorders Week in Chicago, Illinois on October 11, 2018.

Contributions: JSZ: project development, data collection, data analysis, manuscript preparation; SJH: data analysis, manuscript preparation; SA: data analysis; AOZ: project development, data collection, manuscript preparation; YT: project development, data collection, manuscript preparation; JJN: project development, data collection, manuscript preparation; TY: project development, data analysis, manuscript preparation.

Conflict of interest: The authors declare no conflict of interest.

Availability of data and materials: All data generated or analyzed during this study are included in this published article.

Ethics approval and consent to participate: ClinicalTrials.gov identifier: NCT03136601 (https://clinicaltrials.gov/ct2/show/NCT03136 601). All patients participating in this study signed a written informed consent form for participating in this study.

Informed consent: Written informed consent was obtained from a legally authorized representative(s) for anonymized patient information to be published in this article.

Received for publication: 13 May 2020

Revision received: 2 November 2021.

Accepted for publication: 16 November 2021

This work is licensed under a Creative Commons Attribution NonCommercial 4.0 License (CC BY-NC 4.0).

(C) Copyright: the Author(s), 2022

Licensee PAGEPress, Italy

Urogynaecologia 2022; 34:251

doi:10.4081/uij.2022.251

pare quality of life, patient satisfaction, and symptom bother between the monthly maintenance and PRN maintenance groups 
for PTNS therapy. We hypothesized that there is no difference between groups for these three measures.

\section{Materials and Methods}

This was a single-center randomized trial with ethical approval from the Los Angeles Biomedical Research Institute Institutional Review Board. This study was conducted at the Female Pelvic Medicine and Reconstructive Surgery (FPMRS) clinic at Harbor-UCLA Medical Center, a Department of Health Services facility in Los Angeles County, California. Patients were recruited between November 2016 and December 2017.

Women were included if they completed 12 weekly sessions of PTNS for OAB after failing first- and second-line therapy (behavioral therapy and at least two pharmacotherapies) and wished to continue PTNS. All patients had discontinued OAB medication at least 12 weeks prior to study enrollment. Patients were included if they were age 18 years, capable of giving informed consent, spoke primary language of English or Spanish, ambulatory, and capable and willing to follow all studyrelated procedures. Patients were excluded if they did not meet inclusion criteria, if their $\mathrm{OAB}$ was treated with Onabotulinum toxin $A$ in the past year, and if they had a urinary tract infection at time of enrollment. We also excluded prisoners, pregnant women, or women planning to become pregnant during the projected study period.

While we initially encouraged all patients to complete 12 weeks of PTNS treatment, during the study recruitment period our clinic's practice evolved to include an assessment of patient response at the fourth and eighth PTNS visits. Response was assessed with the Patient Global Impression of Improvement (PGI-I) questionnaire. ${ }^{10}$ If patients did not respond "a little better," "much better," or "very much better," they were offered to terminate PTNS and switch to a different OAB treatment. Due to this change in our clinic's practice, some study participants completed 12 PTNS sessions without an interval efficacy assessment, while other participants completed the 12 sessions after the interval assessment was consistent with symptomatic improvement as measured by the PGI-I. Regardless, all study participants wished to continue PTNS after completing the 12 sessions.

Patients were recruited at their $12^{\text {th }}$ PTNS session. No participants concurrently took any pharmacotherapy for OAB treatment. Participants were randomly assigned using a blocked randomized design. Allocation was concealed from participant and research staff until eligibility screening was completed and study condition was assigned. These tasks were completed by trained research team members whose actions were independent of clinical care. A computer-generated randomization sequence, password protected to recruitment staff and participants, was used to assign participants. Although it was not possible to have treatment blinded, all data collection were conducted in blinded fashion independently of the care setting (data was collected by study stuff unaware of the patients' treatment group assignment). All data was stored in REDCap.

The monthly maintenance group had one scheduled appointment per month. The PRN maintenance group called the clinic nurse to schedule PTNS sessions when they felt a return or worsening of their OAB symptoms. The PRN group's maintenance sessions were scheduled anywhere from two to 12 weeks apart.

At enrollment, all patients had a pointof-care urinalysis in order to rule out acute cystitis. At the same visit, patients completed several questionnaires in their preferred language, with the direct assistance of study staff. All questionnaires were previously validated for research and clinical practice in both English and Spanish. Quality of life, the primary outcome, was measured with the Overactive Bladder Questionnaire Short Form (OAB-q SF), which includes a Health-Related Quality of Life (HRQL) Scale. ${ }^{11,12}$ Secondary outcomes included patient satisfaction and degree of symptom bother. Patient satisfaction was measured with both a Visual Analog Scale (VAS) ${ }^{13}$ and the Benefit, Satisfaction and Willingness to Continue (BSW) questionnaire. ${ }^{14,15}$ Symptom bother was assessed with the Overactive Bladder Symptom Score (OAB-SS) ${ }^{16,17}$ as well as the Overactive Bladder Questionnaire Short

What mode of transportation (e.g. car, bus, rideshare) do you use to get to appointments?
What is the length of time you need to get to appointments?
Do you have to take time off work to get to appointments?
Was it difficult to get to the appointment today?
Do you feel satisfied with your treatment?
What is the best part of this treatment?
What is the most challenging part of this treatment?

Figure 1. Patient questionnaire.
Form Symptom Bother Scale (OAB-q SF SBS). ${ }^{11,12}$ Raw scores from the OAB-SS were converted to a percentage so that the English and Spanish versions would be directly comparable, since the English OAB-SS scored symptoms on a scale of 015 and the Spanish OAB-SS scored symptoms on a scale of 0-28. Additionally, patients answered open-ended questions (Figure 1) with direct assistance of study staff. With the exception of the open-ended questions, all of these measures of patientreported outcomes were repeated three months after study enrollment.

Urgent PC Neuromodulation System (Uroplasty, Inc., Minnetonka, MN) was used to deliver retrograde neuromodulation to the sacral nerve plexus with temporary insertion of a 34 gauge needle electrode using a protocol previously described. ${ }^{18}$

An a-priori power analysis for an equivalence test of means determined that sample sizes of 78 in the reference group and 78 in the treatment group were needed to achieve $80 \%$ power at a $5.0 \%$ significance level. Given the limitations for recruiting 158 patients in our clinical setting, the current study is underpowered to determine noninferiority. Therefore, analyses focused on trends and effect size calculations for the current data; cut-offs were set for small ( $\mathrm{g}=$ $0.2)$, medium $(\mathrm{g}=0.5)$, and large $(\mathrm{g}=0.8)$ effect as general guidelines to interpret effect sizes. Generally, effect sizes estimate the magnitude of an effect, or the practical applicability of the statistic.

Statistical analysis was conducted using IBM SPSS Statistics 22. Data were gathered at baseline, three months after enrollment, and six months after enrollment; change scores were calculated for each assessment by subtracting scores obtained at follow-up t-tests were used for comparison of change scores between groups to determine if the proportion of change from baseline is comparable between the two groups. from those obtained at enrollment. Student 
Additionally, paired sample t-tests were calculated separately for the monthly maintenance and PRN maintenance groups to determine if changes from baseline to follow-up were significant.

Qualitative data was also gathered at baseline and three- and six-month month follow-up to assess factors that may impact patients' experiences with their medical care. Some of the questions included selfreported difficulty with mobility (abnormal gait, walker, cane, wheelchair, etc.), average length of time allotted for each appointment, what was the best part and what was most challenging part about the treatment (Figure 1). Qualitative responses were assessed by reviewing all responses to identify trends or common themes across patients.

\section{Results}

Over a 14-month period, 47 patients were assessed for eligibility, 45 met eligibility criteria, and 36 patients were recruited into the study (Figure 2). Patients were randomized in the monthly maintenance $(\mathrm{N}=19)$ or PRN maintenance groups
$(\mathrm{N}=17)$; both groups were similar in demographic characteristics (Table 1). Of note, the majority of patients in both groups were Hispanic and primarily Spanish-speaking. Additionally, the average number of visits to the clinic was not significantly different for the monthly maintenance group (1.79 visits \pm 1.18$)$ compared to the PRN maintenance group (1.24 visits \pm 1.35$),(\mathrm{p}=0.197)$. Although no patients formally switched groups or maintenance schedules, a few patients in the monthly maintenance group routinely missed or rescheduled their appointments. After six months of maintenance therapy, eight $(42 \%)$ patients in the monthly maintenance group and nine (53\%) patients in the PRN maintenance group chose to continue PTNS maintenance treatment ( $\mathrm{p}=0.27$; Table 2).

Patient quality of life was assessed by comparing scores on the OAB-q SF HRQL scale. Questionnaire scores in the monthly maintenance group did not change significantly from baseline (34.87) to three months post-enrollment (32.00, $\mathrm{p}=0.572)$, but quality of life scores did improve significantly from baseline (43.92) to three months post-enrollment $(32.00, \mathrm{p}=0.023)$ in the PRN maintenance group. The change in quality of life between the two groups was also significantly different, with the PRN maintenance group achieving a larger improvement in quality of life over the three-month time period ( $\mathrm{p}=0.039$; Table 3 ).

Patient satisfaction was assessed by comparing scores on the VAS and the BSW questionnaire. The change in VAS score from baseline to three-months post-enrollment was not significantly different in either the monthly $(0.47, \mathrm{p}=0.556)$ or PRN maintenance $(0.97, \mathrm{p}=0.114)$ group. A comparison of change in VAS score over time between the two groups revealed a small effect size $(0.18, \mathrm{p}=0.621)$ which is neither statistically nor clinically significant. Similarly, the change in BSW questionnaire scores over the study period was not significantly different in the monthly (1.07, $\mathrm{p}=0.177$ ) maintenance group. The change in BSW score over three months in the PRN maintenance group showed a small difference (1.40, $\mathrm{p}=0.015)$; however, a comparison of change over time between the two groups revealed a small effect size $(0.13$, $\mathrm{p}=0.715$ ) which is neither statistically nor clinically significant (Table 3). Together, analyses of the VAS score and BSW questionnaire score suggest that patient satisfaction with PTNS is comparable between a monthly and an as-needed maintenance

Table 1. Sample description stratified by allocation group.

\begin{tabular}{lccc} 
& Monthly maintenance (n=19) & PRN maintenance (n=17) & p-value \\
Age (y) & 58.4 & 58.1 & 0.95 \\
Ethnicity Hispanic, Latina, or Spanish origin & $18(94.7)$ & $11(64.7)$ & 0.42 \\
\hline Language & & & 0.01 \\
$\quad$ Spanish & $18(94.7)$ & $11(64.7)$ & \\
English & $1(5.3)$ & 10.5 & 0.09 \\
Education (y) & 7.5 & $13,653.33$ & 0.29 \\
Yearly household income (USD) & $10,002.67$ & 29.8 & 0.75 \\
Years in the USA (y) & 31.4 & & \\
\hline
\end{tabular}

Table 2. Patterns of PTNS follow-up.

Monthly maintenance $(\mathrm{n}=19) \quad$ PRN maintenance $(\mathrm{n}=17) \quad$ p-value

Average number of visits in three months

$1.79 \pm 1.18$

$1.24 \pm 1.35$

0.20

Patients continuing PTNS after six months

$8(42.1)$

$9(52.9)$

0.27

Table 3. Average change in quality of life, patient satisfaction, and symptom bother.

\begin{tabular}{|c|c|c|c|c|c|c|c|c|}
\hline & Baseline & $\begin{array}{l}\text { Monthly } \\
3 \text { months }\end{array}$ & p-value & Baseline & $\begin{array}{l}\text { PRN } \\
\text { 3 months }\end{array}$ & p-value & Effect size* & p-value \\
\hline $\begin{array}{l}\text { Quality of life } \\
\text { OAB-q SF HRQL }\end{array}$ & 34.9 & 32.0 & 0.57 & 43.9 & 32.0 & 0.02 & 0.82 & 0.04 \\
\hline $\begin{array}{l}\text { Patient Satisfaction } \\
\text { VAS } \\
\text { BSW }\end{array}$ & $\begin{array}{l}7.29 \\
8.38\end{array}$ & $\begin{array}{l}6.82 \\
7.31\end{array}$ & $\begin{array}{l}0.56 \\
0.18\end{array}$ & $\begin{array}{l}7.77 \\
9.13\end{array}$ & $\begin{array}{l}6.80 \\
7.73\end{array}$ & $\begin{array}{l}0.11 \\
0.02\end{array}$ & $\begin{array}{l}0.18 \\
0.13\end{array}$ & $\begin{array}{l}0.62 \\
0.72\end{array}$ \\
\hline $\begin{array}{c}\text { Symptom Bother } \\
\text { OAB-SS (\%) } \\
\text { OAB-q SF SBS }\end{array}$ & $\begin{array}{l}63.9 \\
14.4 \\
\end{array}$ & $\begin{array}{l}66.4 \\
17.7 \\
\end{array}$ & $\begin{array}{l}0.60 \\
0.09\end{array}$ & $\begin{array}{l}50.5 \\
16.9 \\
\end{array}$ & $\begin{array}{l}63.1 \\
18.1 \\
\end{array}$ & $\begin{array}{l}0.01 \\
0.69\end{array}$ & $\begin{array}{l}0.55 \\
0.23\end{array}$ & $\begin{array}{l}0.13 \\
0.52\end{array}$ \\
\hline
\end{tabular}

*Effect size comparing change in symptom between groups 
schedule. Changes in patient symptomatology were assessed by comparing OAB-SS as well as scores on the OAB-q SF Symptom Bother Scale. In the monthly maintenance group, OAB-SS remained about the same over the study period, but the score worsened in the PRN maintenance group $(+12.52 \%, \mathrm{p}=0.011)$. The effect size (0.55), however, was moderate and the difference in scores between the two groups was not statistically significant $(\mathrm{p}=0.13)$. There were no significant changes over time in OAB-q SF Symptom Bother Scale in either group, and the difference between the change in score between the groups was moderate (effect size $=0.23$ ) and not statistically significant ( $\mathrm{p}=0.523$; Table 3 ).

In the open-ended questionnaire, patients reported various difficulties in getting to PTNS appointments. Patients reported a wide range (between 30 minutes and eight hours) in how much time they budgeted for their planned 30-minute appointment. There was no difference in how much time patients in the different groups budgeted for appointments: the monthly group budgeted $3.5+/-2.13$ hours and the PRN group budgeted $2.8+/-1.5$ hours $(\mathrm{p}=0.45)$. Reported barriers to making PTNS appointments were often transportation-themed, with reported obstacles such as "I couldn't find a ride," "traffic," and "parking." Taking time off work was another common impediment. Patients often remarked displeasure with how long their PTNS appointments took. When asked about the positive aspects of PTNS maintenance therapy, patients cited symptom improvement in general, symptom improvement as compared to other forms of $\mathrm{OAB}$ treatment, and satisfaction with the clinic staff.

\section{Discussion}

Our study compared monthly to PRN PTNS maintenance and found a difference in patient quality of life, but no significant differences in other patient-reported outcomes such as patient satisfaction with treatment and symptom bother. Quality of life scores significantly improved from baseline in the PRN maintenance group. The PRN maintenance group also achieved a larger improvement in quality of life compared to the difference seen in the monthly maintenance group; this effect size was large (0.82), implying high real-world practicality, and statistically significant. There was a small exception in the trend of no difference of secondary outcomes. Symptom severity as measured by OAB-SS worsened over the three-month study period in the PRN maintenance group. The effect size, however, was moderate and the difference in scores between the two groups was not statistically significant. These mixed findings suggest that analysis of a larger sample size is warranted to assess the impact of PTNS maintenance schedule on patientreported outcomes, especially quality of life and symptom severity.

Although these analyses are underpowered, our findings suggest that there may be no difference between PTNS maintenance schedules and most patient-reported outcomes (patient satisfaction and symptom bother). However, since quality of life seems to be improved in the PRN group, perhaps spacing PTNS maintenance appointments is a reasonable step to optimize care of $\mathrm{OAB}$ patients. The literature already supports less frequent timing of PTNS maintenance: in the STEP study, sub-

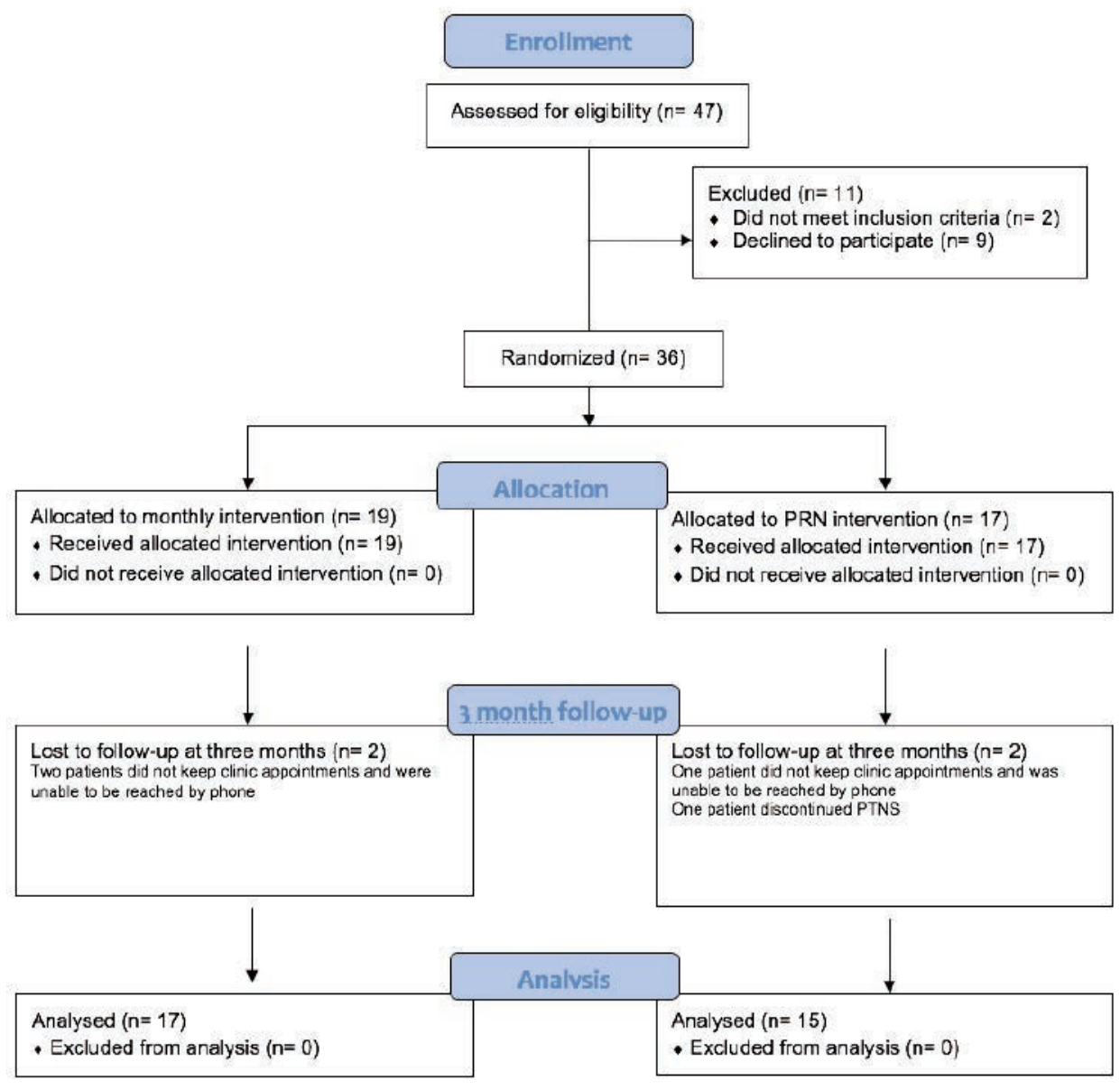

Figure 2. CONSORT diagram. 
jects received PTNS therapy according to a transitional prescribed tapering protocol that included two treatments at two-week intervals, two treatments at three-week intervals, then one treatment at a four-week interval. After the tapering, subjects were put on a personal treatment plan, similar to our PRN maintenance group. ${ }^{19}$ In the STEP study, patients averaged 1.3 PTNS visits per month, while our PRN maintenance group averaged 1.25 visits over a three-month period, all while maintaining satisfaction with treatment.

Our analyses did show an improvement in quality of life scores both in the PRN group over time, and in the PRN group as compared to the monthly maintenance group. Curiously, there was not a significant difference between the groups' average number of visits during the study period. The difference in quality of life between groups therefore is likely due to other factors, such as patient difficulty in attending monthly appointments, or an increased sense of control over one's schedule in the PRN group. In the open-ended questionnaire, many patients shared their frustrations with attending PTNS appointments, such as difficulty with transportation (many of our patients rely on public busses), parking, or appointments running late or taking too much time. Surely there is a financial burden to attending many PTNS appointments as well, as supported by patient frustrations with having to take time off work. From previous research on barriers to incontinence care, we understand that Hispanic patients (who constitute the majority of patients in our study) must overcome more healthcare barriers as compared to women of other races, even when potential confounders such as age, income, and education are adjusted for. ${ }^{20}$

A likely confounder was variability in the monthly maintenance group's appointment schedule. Although no patients formally switched study groups, a number of patients in the monthly maintenance group routinely missed or rescheduled their appointments, effectively somewhat transforming this study into an unplanned crossover study. The reasons for these missed or rescheduled appointments was unclear, but these patients' schedules may have more closely aligned with the PRN maintenance group's, especially if these patients rescheduled based on when they felt a return or worsening of their $\mathrm{OAB}$ symptoms.

Limitations to this study include the small sample size and homogeneous patient population. In addition, study recruitment was hindered by an evolution in how our FPMRS clinic assessed patient response to
PTNS. We initially encouraged all patients to complete 12 weeks of PTNS treatment, but during the study recruitment period we began assessing patient response to PTNS at the fourth and eighth PTNS visits. Response was assessed with the Patient Global Impression of Improvement (PGI-I) questionnaire. ${ }^{10}$ If patients did not respond "a little better," "much better," or "very much better," they were offered to terminate PTNS and switch to a different OAB treatment. Due to this change in our practice, fewer patients completed all 12 weeks of initial PTNS therapy, which was one of our study inclusion criteria. This hinderance in study enrollment is a major reason why patient enrollment was less than originally estimated; additionally, the difference in how PTNS efficacy was measured could be a source of bias. Furthermore, baseline quality of life scores was difference between the two groups; this certainly could be a potential confounder.

Strengths of our study included the randomized trial study design, as well as the focus on Hispanic and Latina women with $\mathrm{OAB}$, who are often underrepresented in FPMRS research but suffer from PFDs at a high rate. The RRISK study, a populationbased study of middle-aged and older women, found that the age-adjusted prevalence of weekly incontinence was highest among Hispanic women, followed by White, Black and Asian-American women. ${ }^{21}$ Our study population differs from the majority of $\mathrm{OAB}$, and more specifically, PTNS research, in that the patients are mostly Hispanic, Spanish speaking, and of low socioeconomic status.

\section{Conclusions}

Assessing patient-reported outcomes is an integral part of evaluating the efficacy of treatments such as PTNS for OAB. Assessing these outcomes in underrepresented populations such as the one studied in this research is all the more important, especially given the high prevalence of UI in this Hispanic population and the especially burdensome barriers to care. Our study suggests that overall there may be neither significant statistical or practical differences in subjective outcomes such as patient satisfaction and symptom bother between scheduled monthly and PRN PTNS maintenance therapy. Although our results suggest a small improvement in patient qualify of life in PRN PTNS therapy, we acknowledge that due to the unplanned crossover design and small number of study participants we cannot make definitive conclusions about how patient- reported $\mathrm{OAB}$ outcomes vary between the PTNS maintenance groups. We can only definitely conclude that keeping PTNS appointments can be very difficult for patients, especially in a low-resource population. A larger study may confirm our findings and reveal an opportunity to optimize OAB therapy.

\section{References}

1. Stewart F, Gameiro OL, El Dib R, et al. Electrical stimulation with nonimplanted electrodes for overactive bladder in adults. Cochrane Database Syst Rev 2016;4:Cd010098.

2. Shamliyan T, Wyman JF, Ramakrishnan $\mathrm{R}$, et al. Benefits and harms of pharmacologic treatment for urinary incontinence in women: a systematic review. Ann Intern Med 2012;156:861-74, W301-10.

3. Gormley EA, Lightner DJ, Faraday M, et al. Diagnosis and treatment of overactive bladder (non-neurogenic) in adults: AUA/SUFU guideline amendment. J Urol 2015;193:1572-80.

4. Staskin DR, Peters KM, MacDiarmid S, et al. Percutaneous tibial nerve stimulation: a clinically and cost effective addition to the overactive bladder algorithm of care. Current Urol Rep 2012;13:32734.

5. Peters KM, Macdiarmid SA, Wooldridge LS, et al. Randomized trial of percutaneous tibial nerve stimulation versus extended-release tolterodine: results from the overactive bladder innovative therapy trial. J Urol 2009; 182:1055-61.

6. Gaziev G, Topazio L, Iacovelli V, et al. Percutaneous Tibial Nerve Stimulation (PTNS) efficacy in the treatment of lower urinary tract dysfunctions: a systematic review. BMC Urol 2013;13:61.

7. van Leeuwen JH, Castro R, Busse M, et al. The placebo effect in the pharmacologic treatment of patients with lower urinary tract symptoms. Eur Urol 2006;50:440-52; discussion 453.

8. Peters KM, Carrico DJ, MacDiarmid SA, et al. Sustained therapeutic effects of percutaneous tibial nerve stimulation: 24-month results of the STEP study. Neurourol Urodynamics 2013;32:24-9.

9. Lee HN, Chae JY, Lee HS, et al. Translation and linguistic validation of the Korean version of the treatment satisfaction visual analogue scale and the overactive bladder satisfaction with treatment questionnaire. Int Neurourol J 2017;21:309-19. 
10. Yalcin I, Bump RC. Validation of two global impression questionnaires for incontinence. Am J Obstet Gynecol 2003;189:98-101.

11. Coyne KS, Thompson CL, Lai JS, et al. An overactive bladder symptom and health-related quality of life short-form: validation of the OAB-q SF. Neurourol Urodyn 2015;34:255-63.

12. Arlandis S, Ruiz MA, Errando C, et al. Quality of life in patients with overactive bladder: validation and psychometric properties of the Spanish Overactive Bladder Questionnaire-short Form. Clinical Drug Invest 2012;32:523-32.

13. Stach-Lempinen B, Kujansuu E, Laippala $\mathrm{P}$, et al. Visual analogue scale, urinary incontinence severity score and 15 D--psychometric testing of three different health-related quality-of-life instruments for urinary incontinent women. Scandinavian J Urol Nephrol
2001;35:476-83.

14. Pleil AM, Coyne KS, Reese PR, et al. The validation of patient-rated global assessments of treatment benefit, satisfaction, and willingness to continue-the BSW. Value Health 2005;8 Suppl 1:S25-34.

15. Jimenez MA, Cambronero J. Validation of the Spanish version of the questionnaire $<<$ Benefit, satisfaction and willingness to continue the treatment $>>$ in patients with overactive bladder. Actas Urologicas Espanolas 2013;37:473-9.

16. Homma Y, Yoshida M, Seki N, et al. Symptom assessment tool for overactive bladder syndrome--overactive bladder symptom score. Urology 2006;68:318-23.

17. Weinberg AC, Brandeis GH, Bruyere J, et al. Reliability and validity of the Overactive Bladder Symptom Score in Spanish (OABSS-S). Neurourol
Urodynam 2012;31:664-8.

18. Peters KM, Carrico DJ, Perez-Marrero RA, et al. Randomized trial of percutaneous tibial nerve stimulation versus Sham efficacy in the treatment of overactive bladder syndrome: results from the SUmiT trial. J Urol 2010;183:143843.

19. Peters KM, Carrico DJ, Wooldridge LS, et al. Percutaneous tibial nerve stimulation for the long-term treatment of overactive bladder: 3-year results of the STEP study. J Urol 2013;189:2194-201.

20. Willis-Gray MG, Sandoval JS, Maynor $\mathrm{J}$, et al. Barriers to urinary incontinence care seeking in White, Black, and Latina women. Female Pelvic Med Reconstruct Surg 2015;21:83-6.

21. Thom DH, van den Eeden SK, Ragins AI, et al. Differences in prevalence of urinary incontinence by race/ethnicity. J Urol 2006;175:259-64. 